\title{
Tenosinovitis estenosante de los dedos de la mano (dedo en gatillo)
}

\author{
Stenosing flexor tenosynovitis of the hand (trigger finger) \\ Jorge Eduardo Molina Ortega, * Carlos Gargollo Orvañanos, \\ Esteban Israel Campos Serna, \$ Mauricio De la Concha Tiznado \\ Citar como: Molina OJE, Gargollo OC, Campos SEI, De la Concha TM. Tenosinovitis estenosante de los dedos \\ de la mano (dedo en gatillo). Acta Med. 2020; 18 (4): 424-426. https://dx.doi.org/10.35366/97273
}

\section{INTRODUCCIÓN}

La tenosinovitis estenosante de los dedos de la mano, o mejor conocida como dedo en gatillo, es una de las principales causas de incapacidad y dolor en la mano. El fenómeno del atrapamiento del tendón se debe al pinzamiento mecánico de los tendones flexores de los dedos cuando pasan a través de una polea retinacular estrecha a la altura de la cabeza del metacarpiano. ${ }^{1-3}$

La forma más frecuente de dedo en gatillo es el tipo primario, el cual se encuentra en mujeres de mediana edad, por lo demás sanas, con una frecuencia dos a seis veces mayor que en hombres. ${ }^{1,2,4,5} \mathrm{El}$ dedo más frecuentemente afectado es el pulgar, seguido del anular, medio, meñique e índice. ${ }^{4}$ Los dedos en resorte de tipo secundario pueden observarse en pacientes con diabetes mellitus, gota, enfermedad renal, artritis reumatoide y otras enfermedades reumáticas. ${ }^{6-8}$

\section{FISIOPATOLOGÍA}

En los dedos de las manos, cada tendón flexor pasa a través de una serie de estrechos canales fibro óseos destinados a optimizar el balance entre el movimiento y la producción
Tabla 1: Datos demográficos $(N=140)$

\begin{tabular}{lc} 
& $n(\%)$ \\
\hline Género & \\
Femenino & $87(62.0)$ \\
Masculino & $53(38.0)$ \\
Edad (años) & \\
0 a 10 & $1(1.0)$ \\
11 a 20 & $0(0)$ \\
21 a 30 & $4(3.0)$ \\
31 a 40 & $6(4.0)$ \\
41 a 50 & $18(13.0)$ \\
51 a 60 & $46(33.0)$ \\
61 a 70 & $41(29.0)$ \\
71 a 80 & $17(12.0)$ \\
81 a 90 & $7(5.0)$ \\
91 a 100 & $0(0)$ \\
Comorbilidades & \\
Sanos & $88(64.0)$ \\
Hipertensión arterial & $16(12.0)$ \\
Hipotiroidismo & $13(8.0)$ \\
Diabetes mellitus & $6(5.0)$ \\
Otros & $5(4.0)$ \\
Dos o más & $12(7.0)$ \\
& \\
\hline
\end{tabular}

* Residente de Alta Especialidad en Procedimientos Avanzados de Mano del Hospital Ángeles Pedregal. Facultad Mexicana de Medicina de la Universidad La Salle. Ciudad de México, México.

₹ Cirujano de Mano del Hospital Ángeles Pedregal. Ciudad de México, México.

\$ Residente de Cirugía Plástica y Reconstructiva. Hospital Central Sur de Alta Especialidad Petróleos Mexicanos. Ciudad de México, México.

www.medigraphic.com/actamedica
" Residente de Cirugía General del Hospital Ángeles Pedregal. Facultad Mexicana de Medicina de la Universidad La Salle. Ciudad de México, México.

Correspondencia:

Dr. Jorge Eduardo Molina Ortega

Correo electrónico: jorge_molort@hotmail.com

Aceptado: 14-04-2020.

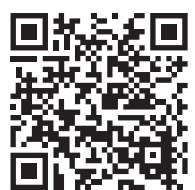


Tabla 2: Tratamiento y seguimiento $(\mathrm{N}=140)$.

\begin{tabular}{|c|c|}
\hline & $\mathrm{n}(\%)$ \\
\hline \multicolumn{2}{|l|}{ Tratamiento } \\
\hline \multicolumn{2}{|l|}{ Médico } \\
\hline $1^{a}$ infiltración & $127(91.0)$ \\
\hline $2^{a}$ infiltración & $44(31.0)$ \\
\hline \multicolumn{2}{|l|}{ Quirúrgico } \\
\hline Percutáneo & $38(27.0)$ \\
\hline Abierto & $8(6.0)$ \\
\hline \multicolumn{2}{|l|}{ Seguimiento (meses) } \\
\hline 0 a 3 & $35(25.0)$ \\
\hline 4 a 12 & $34(24.0)$ \\
\hline 13 a 24 & $32(23.0)$ \\
\hline 25 a 48 & $20(15.0)$ \\
\hline 49 a 180 & $19(13.0)$ \\
\hline
\end{tabular}

de la fuerza para mantener el tendón en aposición cercana a las articulaciones que controla. ${ }^{1-3}$

El fenómeno del atrapamiento del tendón se debe al pinzamiento mecánico de los tendones flexores de los dedos cuando pasan a través de una polea retinacular estrechada a la altura de la cabeza del metacarpiano. ${ }^{1-3}$

Los cambios anatomopatológicos más destacados en las poleas muestran una hipertrofia macroscópica.

Al examen microscópico de las mismas se encuentra degeneración, formación de quistes, división de las fibras e infiltrado de linfocitos y plasmocitos, ${ }^{9}$ así como presencia de condrocitos en la capa más interna o de fricción de las poleas A1 normales y proliferación de los condrocitos y presencia de colágeno tipo III en las poleas engrosadas, es decir, una metaplasia fibrocartilaginosa bajo influencia de las cargas compresivas repetitivas. ${ }^{3}$

El dedo más frecuentemente afectado es el pulgar, seguido por el anular, medio, meñique e índice. ${ }^{5}$

Los dedos en resorte de tipo secundario pueden verse en casos con diabetes mellitus, gota, enfermedad renal, artritis reumatoide y otras enfermedades reumáticas y se asocia a peor pronóstico después de un tratamiento quirúrgico o conservador. ${ }^{7-9}$

La incidencia de dedos en resorte a lo largo de la vida en adultos no diabéticos mayores de 30 años es del 2.2\% y hasta de $10 \%$ entre aquéllos con diabetes mellitus dependientes de insulina. ${ }^{9-11}$

La mayoría de los dedos en resorte primarios pueden ser tratados exitosamente sin cirugía, lo cual coincide con nuestra experiencia, algunos autores documentaron que el tratamiento quirúrgico tiene de un 7 a un 9\% de malos resultados, con complicaciones como lesión nerviosa, infección, dolor en la incisión, deformidad en flexión, deformidad en cuerda de arco del tendón flexor y recurrencia. 1,8 12-14
La inyección de corticoesteroides tiene una frecuencia de éxito muy satisfactoria, particularmente entre los pacientes no diabéticos con compromiso de un solo dedo, un nódulo, un nódulo palpable definido y corta duración de los síntomas. ${ }^{15,16}$

Aunque no se puede asociar una relación entre un corticoesteroide y ruptura de polea, todos los autores recomiendan no practicar inyección de corticoesteroide intratendinoso conociendo el efecto de desgaste de los corticoesteroides en las fibras de colágeno.

Debido a la teoría de la inhibición de la cicatrización, es prudente esperar por lo menos seis semanas después de la inyección de corticoesteroides antes de considerar liberación quirúrgica abierta. ${ }^{8}$

\section{CONCLUSIONES}

A lo largo de estos 10 años, con el uso del algoritmo propuesto por Kerrigan y Stanwix ${ }^{17}$ hemos tratado 140 pacientes, de los cuales sólo 46 requirieron cirugía (Tablas 1 y 2) y el uso del protocolo ha resultado en un adecuado tratamiento para la tenosinovitis estenosante de los dedos de la mano con la ventaja de ser una estrategia clara y fácil de seguir (Anexo 1), además de disminuir costos y aumentar el beneficio para el paciente. Asimismo, contribuye a bajar la tasa de complicaciones que surgen al seguir otras estrategias de tratamiento descritas por otros autores.

\section{REFERENCIAS}

1. Newport ML, Lane LB, Stuchin SA. Treatment of trigger finger by steroid injection. J Hand Surg Am. 1990; 15 (5): 748-750.

2. Patel MR, Bassini L. Dedo en resorte de los dedos y pulgar, cuando ferulizar, inyectar o cirugía. J Hand Surg. 1992; 17 (1): 110-113.

3. Sampson SP, Badalamente MA, Hurst LC. Patología de la polea A1 humana en dedo en resorte. J Hand Surg. 1991; 16 (4): 714-721.

4. Fahey JJ, Bollinger JA. Dedo en resorte en adultos y niños. J Bone Joint Surg. 1954; 36 (6): 1200-1218.

5. Marks MR, Gunther SF. Eficacia de inyección de cortisona en el tratamiento de dedo en resorte. J Hand Surg. 1989; 14 (4): 722-727.

6. Chammas M, Bousquet P, Renard E, Poirier JL, Jaffiol C, Allieu Y. Dupuytren, túnel del carpo, dedo en resorte y diabetes mellitus. J Hand Surg. 1995; 20 (1): 109-114.

7. Griggs SM, Weiss AP, Lane LB. Tratamiento de dedo en resorte en pacientes con diabetes mellitus. J Hand Surg. 1995; 20: 787-789.

8. Stahl S, Kenter Y, Karneilli E. Evolución en el tratamiento de dedo en resorte en diabetes. J Diabetes Complications. 1997; 11 (5): 287-290.

9. Bunnell S. Lesiones de la mano: cirugía de la mano. Philadelphia: J.B. Lippincott; 1979. pp. 496-499.

10. Quinnel RC. Manejo conservador del dedo en resorte. Practitioner. 1980; 224 (1340): 187-190.

11. Eastwood DM, Gupta KJ, Johonson DP. Liberación percutánea de dedo en resorte: procedimiento en consultorio. J Hand Surg. 1992; 17 (1): 114-117.

12. Carrozzella J, Stern PJ, Von Kuster LC. Transección del nervio radial digital del pulgar durante la liberación del resorte. J Hand Surg. 1989; 14: 198-200. 
13. Heithoff SJ, Millender LH, Helman J. Cuerda de arco como complicación de la liberación de dedo en resorte. J Hand Surg. 1988; 13 (4): 567-570.

14. Nagoshi M, Hashizume H, Nishida K. Liberación percutánea de dedo en resorte en pacientes con enfermedad idiopática y en hemodiálisis. Acta Med Okayama. 1997; 51 (3): 155-158.

15. Taras JS, Liams GJ, Gibbons M. Ruptura del flexor largo del pulgar en pulgar en resorte, reporte de caso. J Hand Surg. 1995; 20: 276-227.

16. Fitzgerald BT, Hofmeister EP, Fan RA. Ruptura tardía de los tendones flexores superficial y profundo en dedo en resorte posterior a inyección de corticoesteroides: reporte de caso. J Hand Surg. 2005; 30 (3): 479-482.
17. Kerrigan CL, Stanwix MG. Uso de evidencia para minimizar el costo de manejo de dedo en resorte. J Hand Surg. 2009; 34 (6): 997-1005.

Conflicto de intereses y financiamiento: Jorge Molina, Carlos Gargollo, Esteban Campos y Mauricio De la Concha declaran que no tienen ningún conflicto de intereses. Financiamiento, ninguno.

Anexo 1: Algoritmo de Kerrigan y Stanwix para el tratamiento de la tenosinovitis estenosante de los dedos de la mano.

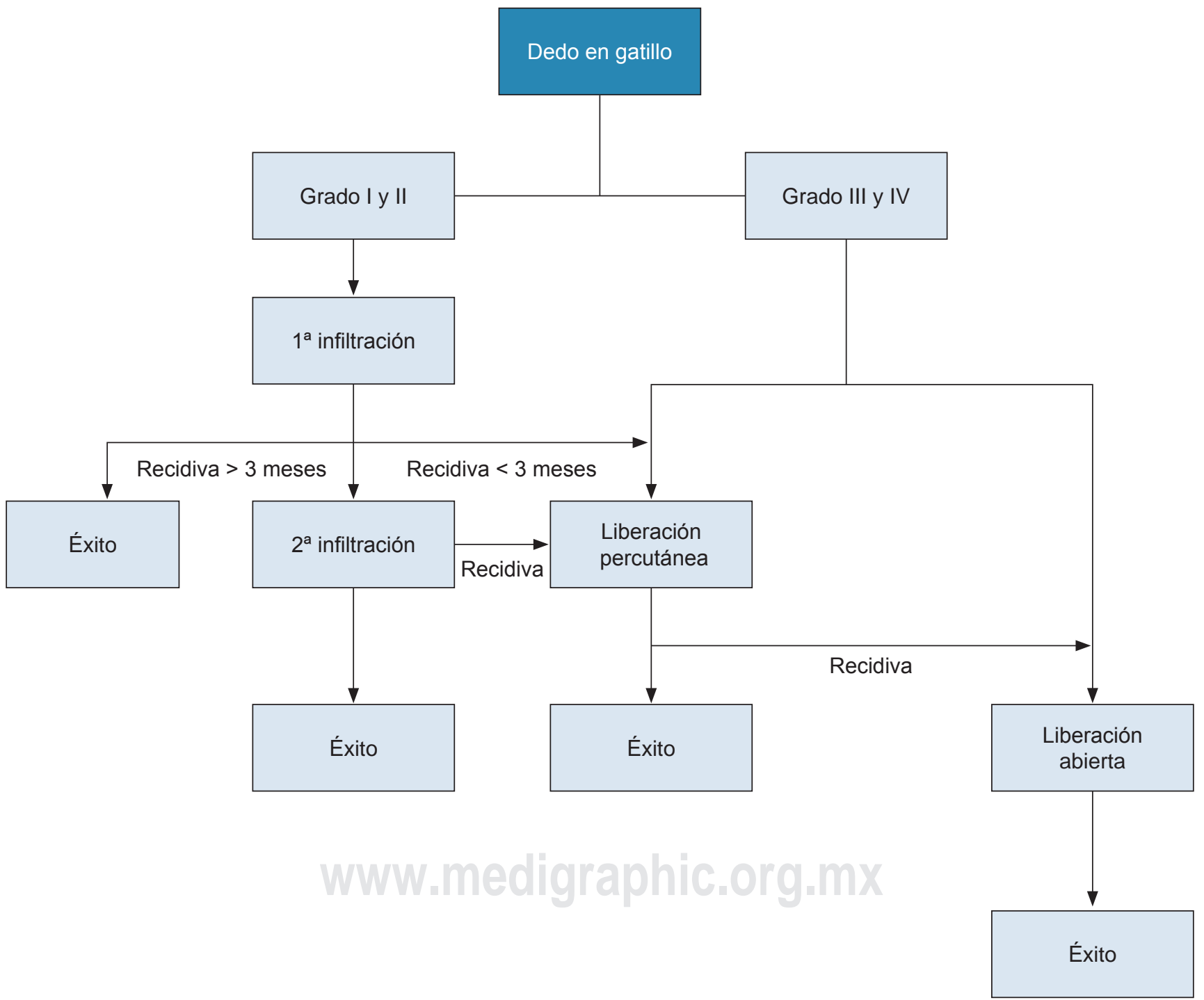

